\title{
Strongyloides stercoralis Infection in the Immunocompromised Host
}

\author{
Roshan Ramanathan, $M D, M P H$, and Thomas B. Nutman, $M D$
}

\begin{abstract}
Corresponding author
Roshan Ramanathan, MD, MPH

Clinical Parasitology Unit and Helminth Immunology Section, Laboratory of Parasitic Diseases, National Institute of Allergy and Infectious Diseases, National Institutes of Health, 4 Center Drive, Building 4, Room B1-05, Bethesda, MD 20892, USA.

E-mail: ramanathanr@niaid.nih.gov
\end{abstract}

Current Infectious Disease Reports 2008, 10:105-110

Current Medicine Group LLC ISSN 1523-3847

Copyright (C) 2008 by Current Medicine Group LLC

Strongyloides stercoralis is an intestinal nematode acquired in the tropics or subtropics. Most often, it causes chronic, asymptomatic infection, but a change in immune status can increase parasite numbers, leading to hyperinfection syndrome, dissemination, and death if unrecognized. Corticosteroid use is most commonly associated with hyperinfection syndrome. Diagnosis of Strongyloides infection is based on serology and serial stool examinations for larvae. The treatment of choice for chronic, asymptomatic infection is oral ivermectin. Alternative pharmacologic agents include albendazole and thiabendazole. For hyperinfection syndrome, ivermectin remains the drug of choice, though therapy duration must be individualized with the end point being complete parasite eradication. Recurrent strongyloidiasis should prompt an evaluation for human T-cell lymphotropic virus type 1 coinfection. No test of cure is currently available, although immunoglobulin $\mathrm{G}$ antibody levels have been shown to decline within 6 months of successful treatment.

\section{Introduction}

Strongyloides stercoralis is an intestinal nematode that infects 3 million to 100 million people worldwide [1]. Although this parasite most commonly causes asymptomatic infection, an alteration in host immune status (eg, corticosteroids) can lead to fatal, fulminant infection. With widespread use of immunosuppressive agents and increased migration from Strongyloides-endemic areas (eg, the tropics and subtropics), numerous opportunities exist for clinicians to encounter hyperinfection syndrome in industrialized countries. Because exposures may be remote and patients unsuspecting, increased awareness among clinicians is critical to identifying and treating this parasite.

\section{Epidemiology}

Although endemic to the tropics and subtropics, foci of infection occur in temperate regions such as Japan, Italy, Australia, and the United States [1]. Within the United States, prevalence rates up to $4 \%$ have been noted in Kentucky and rural Appalachia [2]. Immigrants and refugees comprise a significant population at risk for strongyloidiasis. In one survey, $46 \%$ of Sudanese refugees in the United States had evidence of Strongyloides infection by serology [3•]. Although Strongyloides is most commonly acquired transcutaneously (as discussed in the later section on Life Cycle), several reports raise speculation about alternate routes of transmission [4]. A Japanese study observed a higher prevalence of Strongyloides infection in patients with Blastocystis hominis, a protozoan acquired by the fecal oral route [4]. Personto-person transmission can occur with close contact (eg, kissing), according to one case report [5]. However, standard contact precautions appear to be sufficient to prevent nosocomial transmission [6]. Transmission of Strongyloides infection after transplantation of kidneys, pancreatic allograft, or intestines has been suggested in instances when donors but not recipients had a history of travel to a Strongyloides-endemic area [7-9].

\section{Life Cycle}

Human infection is acquired transcutaneously by contact with infectious filariform larvae in contaminated soil. In the classic pulmonary route, filariform larvae enter the circulation, migrate to the lungs, ascend the tracheobronchial tree, and reach the small intestine after being swallowed. In the small intestine, the larvae molt twice to become adult females that lay eggs in the intestinal mucosa. The eggs hatch into noninfectious rhabditiform larvae that are shed in the stool to become either filariform larvae (direct development) or free-living adult males and females that produce larvae (indirect development). Autoinfection occurs when filariform larvae penetrate the intestinal mucosa or perianal skin to re-enter the circulation and migrate to the lungs.

In the immunocompetent host, cellular immune effector mechanisms and intrinsic parasite biology are believed to regulate the population density of adult worms in 
the intestine [1]. With an altered host immune status (eg, exposure to corticosteroids), even one adult female can multiply rapidly by parthenogenesis, leading to accelerated autoinfection and/or dissemination. The parasite's ability to persist and multiply indefinitely in a host distinguishes $S$. stercoralis from other helminths.

\section{Clinical Manifestations Acute strongyloidiasis}

The clinical manifestations of acute strongyloidiasis can be associated with the path of larval migration from the skin to the small intestine. Infected individuals may experience irritation at the site of skin penetration by larvae, followed by tracheal irritation or dry cough, and ultimately gastrointestinal symptoms (eg, diarrhea, constipation, abdominal pain, anorexia) [10].

\section{Chronic strongyloidiasis}

Chronic strongyloidiasis most frequently causes asymptomatic infection in the immunocompetent individual. Up to $75 \%$ of people may have peripheral eosinophilia or elevated immunoglobulin E levels; therefore, Strongyloides should be considered in the differential diagnosis of high-grade and/or persistent eosinophilia in travelers or expatriates from endemic areas [11•].

Symptomatic individuals may complain of diarrhea, constipation, intermittent vomiting, or borborygmus [10]. Chronic urticaria [12] or larva currens (ie, pruritic linear streaks located along the lower trunk, thighs, and buttocks) may be presenting signs or symptoms of infection. Unusual manifestations of chronic strongyloidiasis include arthritis [13], nephrotic syndrome [14], chronic malabsorption [15], duodenal obstruction [16,17], focal hepatic lesions [18], and recurrent asthma [19].

Hyperinfection syndrome/disseminated strongyloidiasis Hyperinfection syndrome refers to accelerated autoinfection, generally the result of an altered immune status. The distinction between autoinfection and hyperinfection is not strictly defined, but hyperinfection syndrome implies the presence of signs and symptoms attributable to increased larval migration. Development or exacerbation of gastrointestinal and pulmonary symptoms is seen, and increased numbers of larvae in stool and/or sputum is the hallmark of hyperinfection. Disseminated infection occurs when larvae migrate beyond the organs of the autoinfective cycle (lung and gastrointestinal tract), although this may occur at low levels during chronic strongyloidiasis [2]. Untreated, the mortality rate of hyperinfection syndrome and/or disseminated disease approaches $100 \%$ [1].

Hyperinfection syndrome has occurred as late as 65 years after an individual has left a Strongyloides-endemic area $[12,20]$. Eosinophil counts may be normal or even suppressed [10]. Gastrointestinal symptoms commonly occur and may include crampy abdominal pain or bloating, watery diarrhea, constipation, anorexia, weight loss, difficulty swallowing, sore throat, nausea, or vomiting [10]. Diffuse abdominal tenderness and hypoactive bowel sounds may be due to ileus and small bowel obstruction [10]. Protein losing enteropathy can give rise to hypoalbuminemia with peripheral edema and ascites [21]. Mesenteric lymphadenopathy has been reported to cause intestinal pseudo-obstruction in HIV-infected patients with hyperinfection syndrome [22]. Mucosal ulceration can occur in the small intestine as a result of direct invasion of larvae and may be associated with occult blood, hematochezia, or life-threatening gastrointestinal bleeding [23].

Penetration of many larvae through the intestinal wall can be associated with gram-negative sepsis, as larvae carry enteric microorganisms with them into the bloodstream. Recurrent gram-negative sepsis in an individual with history of residence in or travel to an endemic area should prompt consideration of strongyloidiasis. Organisms reported to cause sepsis in such patients include Group D streptococci, Streptococcus bovis meningitis and bacteremia, Escherichia coli, Klebsiella pneumoniae, Proteus mirabilis, Pseudomonas, Enterococcus faecalis, coagulasenegative staphylococci, and Streptococcus pneumonia [10]. Of note, systemic candidiasis has been observed in patients on immunosuppressive regimens [10]. A small, retrospective study suggested that enteric infections-though most commonly associated with disseminated disease-may even occur in chronically infected individuals [2].

Either aseptic or gram-negative meningitis can be associated with disseminated strongyloidiasis [10]. Larvae have been recovered from cerebrospinal fluid, meningeal vessels, dura, epidural, subdural, and subarachnoid spaces [10].

When present, pulmonary manifestations include cough, wheezing, hoarseness, palpitations, atrial fibrillation, pleuritic chest pain, or dyspnea [10]. Petechial hemorrhage, hyperemia of the bronchial mucosa, or, rarely, massive hemoptysis have been reported [10]. Chest radiographs most frequently demonstrate focal or bilateral interstitial infiltrates [10].

Cutaneous periumbilical purpura has been described in patients with disseminated disease due to migration of larvae through vessel walls in the dermis [24]. The syndrome of inappropriate secretion of antidiuretic hormone has also been associated with disseminated infection [25].

\section{Immunocompromised conditions}

\section{Corticosteroids}

For reasons that are not entirely clear, corticosteroids have a particularly strong and specific association with the development of hyperinfection syndrome. Hyperinfection syndrome has been described regardless of dose or route of steroid administration. Even short courses of steroids in immunocompetent patients have led to hyperinfection syndrome and death [26]. Of note, several episodes of disseminated strongyloidiasis have been described with the use of traditional Chinese medications, which contain 
synthetic steroidal compounds [25]. Other therapies or conditions may predispose to dissemination, although the concomitant administration of steroids in most cases makes it difficult to assign a direct causal association (Table 1).

\section{Human T-cell lymphotropic virus type 1 infection}

A growing body of evidence points to the synergistic relationship between human T-cell lymphotropic virus type 1 (HTLV-1) and Strongyloides. Higher rates of Strongyloides infection have been found in HTLV-1/ Strongyloides-coinfected patients. Relapsing Strongyloides infection despite treatment should prompt consideration of HTLV-1 infection [27]. HTLV-1 enhances susceptibility to Strongyloides infection as a result of diminished immunoglobulin E levels and a bias toward a T helper 1 rather than T helper 2 immune response [28]. In turn, Strongyloides may facilitate HTLV-1 virus replication, as suggested by a measurable decline in HTLV-1 messenger RNA levels in one patient after treatment with ivermectin [29]. Strongyloides has been proposed to accelerate the progression of HTLV-1 to adult T-cell leukemia in coinfected patients [30].

\section{HIV}

Hyperinfection syndrome has not been observed frequently with HIV-infected patients despite vast numbers of coinfected individuals. A recent study postulates that lower $\mathrm{CD}^{+}$counts may favor indirect rather than direct development of Strongyloides larvae based on the proportion of free-living adult and infective larvae in stools of coinfected patients [31]. Whether immune reconstitution syndrome occurs after the antiretroviral therapy initiation in Strongyloides-infected patients remains unclear, although case reports have raised this issue [32].

\section{Other conditions}

Case reports have supported an association between Strongyloides infection and primary hypogammaglobulinemia [33]. In such cases, refractoriness to anthelmintic therapy has been noted [10]. Hematologic malignancies such as lymphoma have been associated with hyperinfection syndrome in the absence of corticosteroid use [10]. Relatively few cases of infection following bone marrow transplantation have been reported (Table 1) [10].

\section{Diagnosis}

In chronically infected, asymptomatic individuals, strongyloidiasis diagnosis can be challenging. Definitive diagnosis relies on detection of larvae in the stool. However, intermittent and scanty excretion of larvae limits the utility of stool studies. Diagnostic sensitivity can improve to $100 \%$ when seven stool samples are studied [34]. Various investigators have attempted to improve the diagnostic yield of stool exams using techniques such as direct smear of feces in saline/Lugol's iodine stain, Baermann concentration, Harada-Mori filter paper culture, quantitative formalin

\begin{tabular}{|c|c|}
\hline \multicolumn{2}{|l|}{ Therapies* } \\
\hline Corticosteroids & Doxorubicin \\
\hline Vinca alkaloids & Daunorubicin \\
\hline Azathioprine & Ifosfamide \\
\hline Cyclophosphamide & Melphalan \\
\hline Antithymocyte globulin & Carmustine \\
\hline Anti-CD3 & VP16 \\
\hline Chlorambucil & Mitoxantrone \\
\hline 6-mercaptopurine & Total body irradiation \\
\hline Methotrexate & Etanercept \\
\hline \multicolumn{2}{|l|}{ Bleomycin } \\
\hline \multicolumn{2}{|l|}{ Diseases } \\
\hline HTLV-1 & Malnutrition \\
\hline Hypogammaglobulinemia & $\begin{array}{c}\text { Hematologic malignancies } \\
\text { (eg, lymphoma) }\end{array}$ \\
\hline \multicolumn{2}{|c|}{$\begin{array}{l}\text { *Glucocorticoids were administered concurrently with many } \\
\text { of the drugs listed in this table, and attributing hyperinfection } \\
\text { syndrome to any of these drugs alone is difficult. } \\
\text { HTLV-1- human T-cell lymphotropic virus type } 1 \text {. }\end{array}$} \\
\hline
\end{tabular}

ethyl acetate concentration technique, and nutrient agar plate cultures [34]. Of these, the agar plate culture has been found to be the most sensitive, even in immunocompromised patients. Using this method, larvae could be detected in approximately $50 \%$ of stool samples from individuals with antibody responses to Strongyloides antigen [35]. Duodenal aspiration is more sensitive than stool examination, but it is an invasive procedure, which makes it a less favorable option. Duodenal biopsy can demonstrate parasites nested in the gastric crypts or duodenal glands, as well as eosinophil infiltration of the lamina propria [36].

Enzyme linked immunosorbent assay (ELISA) has been increasingly used in conjunction with stool studies to increase diagnostic sensitivity. The high negative predictive value of the ELISA can be particularly useful in excluding strongyloidiasis as part of the differential diagnosis. Despite its usefulness, serodiagnosis has several limitations, including cross-reactivity in patients with active filarial infections, lower sensitivity in patients with hematologic malignancies or HTLV-1 infection, and inability to distinguish between current and past infection [34]. In addition, the current ELISA relies on the labor-intensive preparation of larval antigen from stool samples of heavily infected humans or experimentally infected animals. Various techniques have been developed in an effort to improve on the drawbacks of serologic-based assays. Recombinant antigens (eg, the NIE antigen) have been proposed as a convenient alternative to the crude antigen currently used [37]. Recently, a dipstick assay was found to be easily and quickly performed and correlated well with ELISA results [38]. A gelatin particle agglutination test has been proposed as an alternative to 


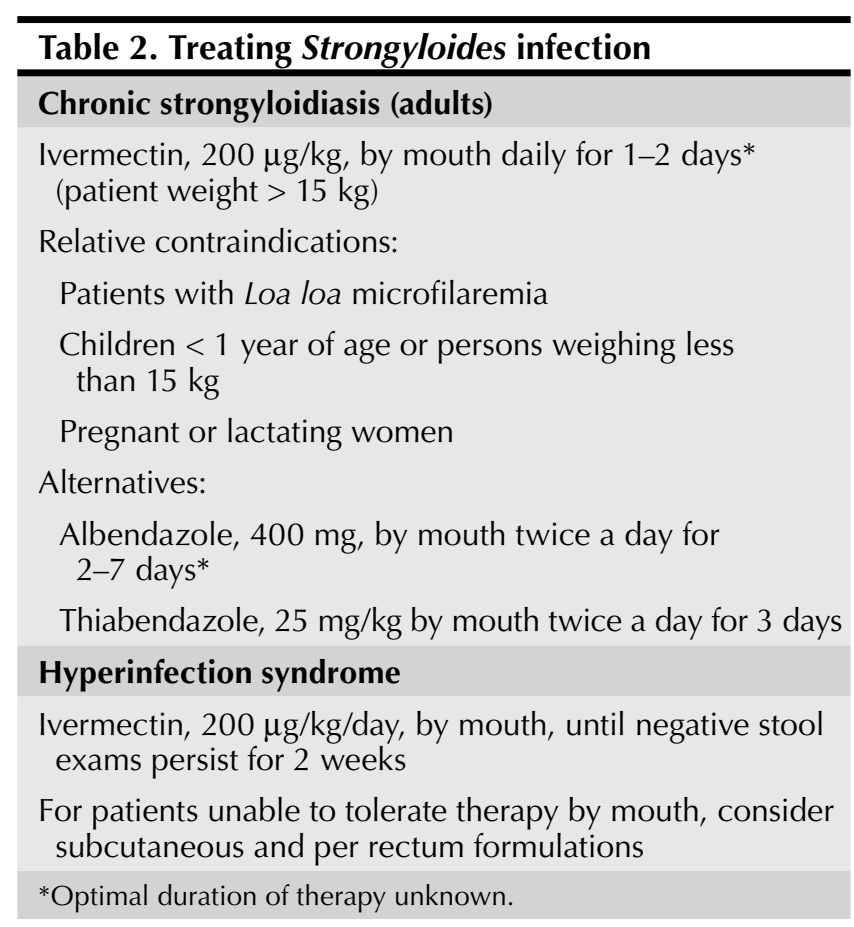

ELISA for mass screening in endemic areas because of its low cost and convenience [39]. An immediate hypersensitivity skin test has been used in a research setting quite effectively, although it may have limited utility in HTLV1 -infected patients [40].

In hyperinfection syndrome, parasitologic diagnosis is simplified by the presence of many larvae in stool and sputum. Larvae have been incidentally found in blood smears, ascitic fluid, and bronchoalveolar lavage specimens [10].

\section{Treatment}

To prevent the development of hyperinfection syndrome, chronically infected, asymptomatic individuals must be treated. Because even one remaining adult female can multiply and cause disseminated disease, the goal of treatment is complete eradication of the parasite. The current treatment of choice for chronic strongyloidiasis is single-dose ivermectin (Table 2), although some studies have suggested that two doses of ivermectin, $200 \mu \mathrm{g} / \mathrm{kg}$, given on consecutive days may have greater efficacy [41]. Ivermectin has better tolerability compared with thiabendazole [42]. In a randomized trial, up to $95 \%$ of patients on thiabendazole experienced side effects, compared with $18 \%$ of ivermectin-treated patients [43]. Side effects of thiabendazole include general fatigue, dizziness, headache, nausea, anorexia, abdominal pain, liver dysfunction, and neuropsychiatric symptoms [43]. Ivermectin has superior efficacy when compared with albendazole, the cure rates of which range from $45 \%$ to $77 \%[42,44]$. Decreased cure rates have been observed in patients with HTLV-1 coinfection regardless of treatment regimen [28]. The US
Centers for Disease Control and Prevention has recommended presumptive predeparture treatment of refugees at risk for Strongyloides [3•]. In such cases, ivermectin should not be given to individuals from Western and Central Africa in whom coinfection with Loa loa has not been excluded. High levels of L. loa microfilaremia can precipitate life-threatening encephalopathy in patients treated with ivermectin. The presumptive use of albendazole must be weighed against the risk of exacerbating inflammatory reactions in patients with neurocysticercosis or those from parasite-endemic areas with a seizure history of unknown etiology.

For disseminated strongyloidiasis, oral ivermectin should be given daily until stool examinations are negative for at least 2 weeks (the duration of the autoinfective cycle) or longer if patients remain immunosuppressed. Off-label rectal administration of ivermectin or thiabendazole, though useful in some critically ill patients [45], can be problematic in patients with severe diarrhea. Patients with paralytic ileus can have difficulty absorbing oral ivermectin due to tissue edema, larger volume of distribution, and increased clearance of unbound drug. Lower serum ivermectin levels than those in normal subjects after oral administration have been demonstrated in

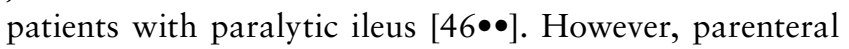
formulations of ivermectin are not approved currently for use in humans; they have only been administered under compassionate use Investigational New Drug exemptions. Experience with subcutaneous ivermectin is based on a few case reports of improved serum drug levels with this route. However, faster drug clearance and lower steady state concentrations were also observed, presumably due to severe hypoalbuminemia, because ivermectin is highly protein bound $[46 \bullet \bullet$.

Despite treatment, fatal progression to acute respiratory distress syndrome can occur even with successful eradication of larvae [20]. Several questions remain unanswered with respect to the treatment of critically ill patients with hyperinfection syndrome. For instance, the ideal dose duration and route of therapy for hyperinfection syndrome has not been studied systematically. Whether to continue or decrease corticosteroid dose in critically ill patients also remains unclear.

Although no test of cure currently exists, serology response by ELISA after treatment can be a useful tool in monitoring treatment response, because antibody levels have been shown to decrease significantly within 6 months after anthelmintic therapy [47]. Serial stool examinations remain the gold standard to assess cure (Table 2).

\section{Prevention}

For persons living in endemic areas, foot protection should be emphasized to prevent cutaneous transmission of the disease. No protective vaccine currently exists. However, a recent animal study demonstrated that DNA immuniza- 
tion with $\mathrm{Na}+\mathrm{K}+\mathrm{ATPase}$ induced protective immunity to challenge with Strongyloides larvae in mice [48].

Mass treatment programs have been proposed as a solution to the challenges of identifying chronically infected Strongyloides patients at risk for hyperinfection syndrome. A small unblinded, uncontrolled study in a Brazilian community demonstrated a $94 \%$ reduction in prevalence following treatment with ivermectin that was sustained for 9 months [49].

\section{Conclusions}

Although S. stercoralis often causes chronic and clinically asymptomatic infection, parasite number can increase substantially in the immunocompromised host, leading to hyperinfection, dissemination, and death if unrecognized. To prevent this, all chronically infected, asymptomatic persons should be treated. Patients from Strongyloidesendemic areas taking corticosteroids must be considered high risk for hyperinfection syndrome, even years after leaving the endemic area. The current method for diagnosis includes serology and serial stool studies for larvae, though limitations of serology include poor specificity and inability to distinguish past from current infection. Nevertheless, the entire diagnostic armamentarium should be brought to bear on a patient with presumptive strongyloidiasis with an eye toward treatment to prevent the often serious sequelae associated with hyperinfection syndrome.

\section{Acknowledgment}

This work was supported by the Division of Intramural Research, National Institutes of Allergy and Infectious Diseases.

\section{Disclosures}

The authors have reported no conflicts of interest relevant to this article.

\section{References and Recommended Reading}

Papers of particular interest, published recently, have been highlighted as:

- Of importance

$\bullet \quad$ Of major importance

1. Genta RM: Strongyloidiasis. In Tropical Infectious Diseases. Edited by Guerrant RL. Philadelphia: Elsevier; 2005:1274-1282.

2. Al-Hasan MN, McCormick M, Ribes JA: Invasive enteric infections in hospitalized patients with underlying strongyloidiasis. Am J Clin Pathol 2007, 128:622-627.

3.- Posey DL, Blackburn BG, Weinberg M, et al.: High prevalence and presumptive treatment of schistosomiasis and strongyloidiasis among African refugees. Clin Infect Dis 2007, 45:1310-1315.

Authors summarized the results of an investigation by the US Centers for Disease Control and Prevention of the prevalence of Strongyloides in a group of refugees from Sudan.
4. Hirata T, Nakamura H, Kinjo N, et al.: Prevalence of Blastocystis hominis and Strongyloides stercoralis infection in Okinawa, Japan. Parasitol Res 2007, 101:1717-1719.

5. Czachor JS, Jonas AP: Transmission of Strongyloides stercoralis person to person. J Travel Med 2000, 7:211-212.

6. Sugiyama K, Hasegawa Y, Nagasawa T, Hitomi S: Exposure of medical staff to Strongyloides stercolaris from a patient with disseminated strongyloidiasis. J Infect Chemother 2006, 12:217-219.

7. Patel G, Arvelakis A, Sauter BV, et al.: Strongyloides hyperinfection syndrome after intestinal transplantation. Transpl Infect Dis 2007, [Epub ahead of print].

8. Ben-Youssef R, Baron P, Edson F, et al.: Stronglyoides stercoralis infection from pancreas allograft: case report. Transplantation 2005, 80:997-998.

9. Said T, Nampoory MR, Nair MP, et al.: Hyperinfection strongyloidiasis: an anticipated outbreak in kidney transplant recipients in Kuwait. Transplant Proc 2007, 39:1014-1015.

10. Keiser PB, Nutman TB: Strongyloides stercoralis in the Immunocompromised Population. Clin Microbiol Rev 2004, 17:208-217.

11. Nutman TB: Evaluation and differential diagnosis of marked, persistent eosinophilia. Immunol Allergy Clin North Am 2007, 27:529-549.

A useful approach to the evaluation of patients with high-grade eosinophilia.

12. Leighton PM, MacSween HM: Strongyloides stercoralis. The cause of an urticarial-like eruption of 65 years' duration. Arch Intern Med 1990, 150:1747-1748.

13. Richter J, Muller-Stover I, Strothmeyer H, et al.: Arthritis associated with Strongyloides stercoralis infection in HLA B-27-positive African. Parasitol Res 2006, 99:706-707.

14. Hsieh YP, Wen YK, Chen ML: Minimal change nephrotic syndrome in association with strongyloidiasis. Clin Nephrol 2006, 66:459-463.

15. Atul S, Ajay D, Ritambhara N, Harsh M, et al.: An unusual cause of malabsorption in an immunocompetent host. J Ayub Med Coll Abbottabad 2005, 17:85-86.

16. Suvarna D, Mehta R, Sadasivan S, et al.: Infiltrating Strongyloides stercoralis presenting as duodenal obstruction. Indian J Gastroenterol 2005, 24:173-174.

17. Harish K, Sunilkumar R, Varghese T, et al.: Strongyloidiasis presenting as duodenal obstruction. Trop Gastroenterol 2005, 26:201-202.

18. Gulbas Z, Kebapci M, Pasaoglu O, Vardareli E: Successful ivermectin treatment of hepatic strongyloidiasis presenting with severe eosinophilia. South Med J 2004, 97:907-910.

19. Tullis DC: Bronchial asthma associated with intestinal parasites. N Engl J Med 1970, 282:370-372.

20. Hauber HP, Galle J, Chiodini PL, et al.: Fatal outcome of a hyperinfection syndrome despite successful eradication of Strongyloides with subcutaneous ivermectin. Infection 2005, 33:383-386.

21. Yoshida H, Endo H, Tanaka S, et al.: Recurrent paralytic ileus associated with strongyloidiasis in a patient with systemic lupus erythematosus. Mod Rheumatol 2006, 16:44-47.

22. Ramdial PK, Hlatshwayo NH, Singh B: Strongyloides stercoralis mesenteric lymphadenopathy: clue to the etiopathogenesis of intestinal pseudo-obstruction in HIVinfected patients. Ann Diagn Pathol 2006, 10:209-214.

23. Csermely L, Jaafar H, Kristensen J, et al.: Strongyloides hyper-infection causing life-threatening gastrointestinal bleeding. World J Gastroenterol 2006, 12:6401-6404.

24. Salluh JI, Bozza FA, Pinto TS, et al.: Cutaneous periumbilical purpura in disseminated strongyloidiasis in cancer patients: a pathognomonic feature of potentially lethal disease? $\mathrm{Braz} J$ Infect Dis 2005, 9:419-424.

25. Seet RC, Gong LL, Tambyath PA: Image of the month. Strongyloides stercoralis hyperinfection and syndrome of inappropriate secretion of antidiuretic hormone. Gastroenterology 2005, 128:8, 252. 
26. Ghosh K, Ghosh K: Strongyloides stercoralis septicaemia following steroid therapy for eosinophilia: report of three cases. Trans R Soc Trop Med Hyg 2007, 101:1163-1165.

27. Hirata T, Uchima N, Kishimoto K, et al.: Impairment of host immune response against Strongyloides stercoralis by human T cell lymphotropic virus type 1 infection. Am J Trop Med Hyg 2006, 74:246-249.

28. Satoh M, Toma H, Sato Y, et al.: Reduced efficacy of treatment of strongyloidiasis in HTLV-I carriers related to enhanced expression of IFN-gamma and TGF-beta1. Clin Exp Immunol 2002, 127:354-359.

29. Ratner L, Grant C, Zimmerman B, et al.: Effect of treatment of Strongyloides infection on HTLV-1 expression in a patient with adult T-cell leukemia. Am J Hematol 2007, 82:929-931.

30. Gabet AS, Mortreux F, Talarmin A, et al.: High circulating proviral load with oligoclonal expansion of HTLV-1 bearing T cells in HTLV-1 carriers with strongyloidiasis. Oncogene 2000, 19:4954-4960.

31. Viney ME, Brown M, Omoding NE, et al.: Why does HIV infection not lead to disseminated strongyloidiasis? J Infect Dis 2004, 190:2175-2180.

32. Currie BJ, McCarthy JS: Strongyloides stercoralis infection as a manifestation of immune restoration syndrome? Clin Infect Dis 2005, 40:635.

33. Seet RC, Lau LG, Tambyah PA: Strongyloides hyperinfection and hypogammaglobulinemia. Clin Diagn Lab Immunol 2005, 2:680-682.

34. Siddiqui AA, Berk SL: Diagnosis of Strongyloides stercoralis infection. Clin Infect Dis 2001, 33:1040-1047.

35. Sato Y, Kobayashi J, Toma H, Shiroma Y: Efficacy of stool examination for detection of Strongyloides infection. $\mathrm{Am} \mathrm{J}$ Trop Med Hyg 1995, 53:248-250.

36. Rivasi F, Pampiglione S, Boldorini R, Cardinale L: Histopathology of gastric and duodenal Strongyloides stercoralis locations in fifteen immunocompromised subjects. Arch Pathol Lab Med 2006, 130:1792-1798.

37. Ravi V, Ramachandran S, Thompson RW, et al.: Characterization of a recombinant immunodiagnostic antigen (NIE) from Strongyloides stercoralis L3-stage larvae. Mol Biochem Parasitol 2002, 125:73-81.

38. van Doorn HR, Koelewijn R, Hofwegen H, et al.: Use of enzyme-linked immunosorbent assay and dipstick assay for detection of Strongyloides stercoralis infection in humans. J Clin Microbiol 2007, 45:438-442.
39. Sithithaworn J, Sithithaworn P, Janrungsopa T, et al.: Comparative assessment of the gelatin particle agglutination test and an enzyme-linked immunosorbent assay for diagnosis of strongyloidiasis. J Clin Microbiol 2005, 43:3278-3282.

40. Neva FA, Gam AA, Maxwell C, Pelletier LL: Skin test antigens for immediate hypersensitivity prepared from infective larvae of Strongyloides stercoralis. Am J Trop Med Hyg 2001, 65:567-572.

41. Igual-Adell R, Oltra-Alcaraz C, Soler-Company E, et al.: Efficacy and safety of ivermectin and thiabendazole in the treatment of strongyloidiasis. Expert Opin Pharmacother 2004, 5:2615-2619.

42. Toma H, Sato Y, Shiroma Y, et al.: Comparative studies on the efficacy of three anthelminthics on treatment of human strongyloidiasis in Okinawa, Japan. Southeast Asian J Trop Med Public Health 2000, 31:147-151.

43. Gann PH, Neva FA, Gam AA: A randomized trial of singleand two-dose ivermectin versus thiabendazole for treatment of strongyloidiasis. J Infect Dis 1994, 169:1076-1079.

44. Marti H, Haji HJ, Savioli L, et al.: A comparative trial of a single-dose ivermectin versus three days of albendazole for treatment of Strongyloides stercoralis and other soil-transmitted helminth infections in children. Am J Trop Med Hyg 1996, 55:477-481.

45. Boken DJ, Leoni PA, Preheim LC: Treatment of Strongyloides stercoralis hyperinfection syndrome with thiabendazole administered per rectum. Clin Infect Dis 1993, 16:123-126.

46.• Turner SA, Maclean JD, Fleckenstein L, Greenaway C: Parenteral administration of ivermectin in a patient with disseminated strongyloidiasis. Am J Trop Med Hyg 2005, 73:911-914.

A description of the experience with off-label use of subcutaneous ivermectin in a critically ill patient.

47. Karunajeewa H, Kelly H, Leslie D, et al.: Parasite-specific IgG response and peripheral blood eosinophil count following albendazole treatment for presumed chronic strongyloidiasis. J Travel Med 2006, 13:84-91.

48. Kerepesi LA, Keiser PB, Nolan TJ, et al.: DNA immunization with $\mathrm{Na}+\mathrm{K}+\mathrm{ATPase}$ (Sseat-6) induces protective immunity to larval Strongyloides stercoralis in mice. Infect Immun 2005, 73:2298-2305.

49. Heukelbach J, Winter B, Wilcke T, et al.: Selective mass treatment with ivermectin to control intestinal helminthiases and parasitic skin diseases in a severely affected population. Bull World Health Organ 2004, 82:563-571. 